\title{
Frosted Branch Angiitis Diagnosed as Neuro-Behçet: A Diagnostic and Etiologic Dilemma
}

\author{
Alejandro Portero J.M. Herreras \\ Ophthalmology Department, University Hospital of Valladolid and IOBA, \\ Valladolid, Spain
}

\section{Key Words}

Frosted branch angiitis · Neuro-Behçet - Uveitis · Behçet's disease

\begin{abstract}
Purpose: To report a case of frosted branch angiitis (FBA) secondary to neuro-Behçet. Methods: Description, diagnosis, angiogram imaging and follow-up of a 28-year-old female with FBA.
\end{abstract}

Results: 'Frosted branch angiitis' is a clinical term applied to three conditions: infiltration of vessels by malignant cells, and sheathing of vessels either secondary to an active disorder or subsequently to a previous inflammatory disease. Our patient's history of two optic neuropathies and the lack of demyelinating signs in neuroimaging made us consider FBA in the context of neuro-Behçet.

Conclusion: Recognition of the category of FBA from the clinical signs is essential to establish the correct diagnosis and prescribe the appropriate treatment.

\section{Introduction}

Frosted branch angiitis (FBA) has been classically considered a disorder characterized by acute panuveitis with vasculitis. FBA onset is sudden and patients complain of painless vision loss, floaters and photopsias. Severe vascular sheathing, as seen on a fundus image, is characteristic of this disorder. The vascular inflammation resembles frosted branches of a tree in winter. It is generally associated with retinal edema and deep retinal hemorrhages. Since its first description in 1976 [1], the term 'frosted branch angiitis' has been indistinctly used in the literature to describe different conditions where fundus imaging discloses an unusual thick sheathing surrounding the retinal vessels. 


\section{Clinical Case}

A 28-year-old female presented to our Department complaining of progressive visual loss in both eyes (OU) for 2 days. No pain or red eye were stated. She had a personal history of anterior ischemic optic neuropathy in the right eye (OD) 5 years ago. Neuroimaging and lab-works done subsequently did not reveal any associated pathology.

The ophthalmic examination disclosed decreased visual acuities (VA) OU: 20/200 OD and 20/120 in the left eye (OS). Ocular motility and pupil function were normal. The intraocular pressure OU was normal along the process. On slit-lamp examination, inflammation was seen in the anterior chamber (cells 2+) and in the vitreous (cells 2+) OU. Fundus examination showed severe sheathing of retinal vessels, retinal hemorrhages, Roth's spots and macular edema OU (fig. 1).

The systemic review only highlighted history of skin folliculitis and recurrent aphthous ulcers. The intense and acute flare of vasculitis led us to consider a case of FBA, and a set of proofs were requested.

Complete blood count, biochemical analysis, and urine test were within normal limits. Angiotensinconverting enzyme and liver function tests were also normal. Serology for hepatitis B and C viruses, human immunodeficiency virus (HIV), syphilis, cytomegalovirus (CMV), Epstein-Barr virus (EBV), toxoplasma and Mantoux test were negative as well as anti-nuclear and anti-neutrophil cytoplasmic antibodies. HLA B51 was positive. Next, computerized tomography (CT) and magnetic resonance imaging (MRI) were requested to rule out involvement of the central nervous system and demyelinating disease, respectively. Neither did disclose any abnormality. Finally, the pathergy test was performed and was positive (erythematous papules $3.5 \mathrm{~mm} 48 \mathrm{~h}$ after the prick).

The suspected diagnosis was FBA secondary to Behçet's disease and neuroimaging did not demonstrate demyelinating signs. The patient started with prednisone $1 \mathrm{mg} / \mathrm{kg}$ p.o., cyclosporine 100 mg t.i.d. p.o. (weight: $65 \mathrm{~kg}$, cyclosporine dose: $4.6 \mathrm{mg} / \mathrm{kg} /$ day), dexamethasone eye drops each $2 \mathrm{~h}$ and homatropine eye drops t.i.d.

Only $24 \mathrm{~h}$ after starting steroids, the inflammation of the vessels improved considerably (fig. 1). Improvement was also seen on fluorescein angiography (FA) which disclosed macular edema OU, no vasculitis, and late peripapillary staining 1 day after the inflammation started.

Seven days after the regimen started, the patient remained with the same VA OU, but the inflammation enhanced both in the anterior chamber (cells $0.5+$ ) and in the vitreous (cells $0.5+$ ). Moreover, funduscopy disclosed a macular star, temporal atrophy on the optic nerve and temporal peripheral hemorrhages associated with exhausted vessels at that level OU (fig. 1).

The patient was kept on that regimen until 3 weeks later when steroids were tapered slowly during 3 months. Four months after her problems started, the patient presented a significant VA improvement (OD 20/20 and OS 20/20) and no intraocular inflammation. Ocular fundus imaging disclosed some hard exudates where macular stars were and mottling of the retinal pigment epithelium at the fovea level. FA only showed an ischemic zone in the temporal retina which produced a peripheral scotoma in the visual fields performed (ig. 2 ).

The patient remained relapse-free for over 1 year, then immunomodulatory therapies (IMTs) were slowly tapered. However, after 9 months when she was on $100 \mathrm{mg}$ a day of that regimen, decreased vision and scotomas OD were noted. After a complete examination and imaging tests, she was diagnosed with ischemic posterior optic neuropathy. The patient started again with steroids, and cyclosporine was increased until $300 \mathrm{mg}$ a day, which totally restored VA in 7 days. MRI keeps on presenting no demyelinating signs. Thus, our patient is currently diagnosed with and followed-up like a case of neuro-Behçet.

\section{Discussion}

FBA is a rare disorder characterized by acute panuveitis associated with vasculitis. Ito et al. [1] first described this disorder in 1976 in a 6-year-old boy with severe bilateral 
white sheathing of all retinal vessels, which inspired the term 'frosted branch angiitis'. Just around 60 cases worldwide [3] have been published up to date.

FBA is usually bilateral and slightly more frequent in females. The prevalence of this disorder depends on the country: in Japan affected patients are usually aged 6-16 years. On the other hand, the reports on cases published from other countries, mostly Caucasian individuals, described a late onset of this disorder (usually in the third decade) [3].

There are several agents and disorders which have been postulated to be causal agents or triggering factors of this entity (table 1) [3]. However, the mechanisms of pathophysiology still remain unknown. The term 'frosted branch angiitis' became confusing over time because it was used to denote an specific disorder as well as just a kind of funduscopic image.

The term remained confusing until 1997 when Kleiner [2] postulated a new differentiation and classification. He divided the term 'frosted branch angiitis' in three categories. The first one, 'frosted branch appearance', denotes a funduscopic image in entities such as leukemia and lymphoma. In these disorders, fake white vessel sheathing simulating a real vessel inflammation can be seen. This appearance is due to infiltration of the vessel walls by malignant cells and not by an inflammatory process.

The second category of FBA is called 'frosted branch response', in which a real vasculitis secondary to active infections or autoimmune diseases is observed (table 1). The third category is 'acute idiopathic frosted branch angiitis', which affects healthy young individuals who have had a infectious precedent which is held capable of causing a subsequent vascular inflammation. This precedent responsible for triggering that secondary vasculitis usually is a flu-like illness, but hepatitis B vaccination, mumps, swine flu vaccination, and bacterial infection can also be responsible for initiating the inflammatory process [3]. Therefore, a hypersensitivity to microbial antigens is the hypothesis most accepted in this subtype of FBA.

Steroid regimen is the most-used treatment in FBA cases with real inflammation [3]. This therapy enhances the vasculitic process a lot (fig. 1). If a specific causal agent is found, it will have to be treated at the same time. On the other hand, if an autoimmune disease is the cause of the FBA, IMTs will be the first choice.

According to Kleiner's classification, our case would match the frosted branch response category. Skin folliculitis, recurrent aphthous ulcers, positive pathergy and HLA B51 suggest the possibility of an underlying Behçet's disease. Furthermore, the patient met the requirements according to the International Criteria for the Diagnosis of Behçet's Disease [4] and cases secondary to this disease have been published [5]. In fact, the two cases of optic neuropathy support the theory of neuro-Behçet. The second flare of neuritis when cyclosporine was tapered would also support the diagnosis. Moreover, there have already been cases of optic neuritis in neuro-Behçet $[6,7]$. On the other hand, the typical neurologic manifestations and neuroimaging signs of neuro-Behçet [8] were not noted, nor that of multiple sclerosis. Therefore, our patient will be monitored closely and biologic therapies are, up to the present, stopped. The patient is currently on $300 \mathrm{mg}$ of cyclosporine a day (weight: $65 \mathrm{~kg}$, dose: $4.6 \mathrm{mg} / \mathrm{kg} /$ day) and is asymptomatic again.

Two other cases of FBA secondary to Behçet's disease have already been published [9, 10]. One case was treated with oral prednisolone and colchicine, which proved effective to 
control the inflammation [9]. The other case was a pregnant patient in whom it was not possible to use any immunomodulatory therapy and where the disease spontaneously resolved [10]. In our case we could have used other IMTs but we decided to use cyclosporine because we had had good results with it in Behçet cases.

In summary, recognition of the correct category of FBA from the clinical signs is essential to prescribe appropriate treatment. That is, in frosted branch appearance cases, therapeutic procedures to treat the malignant disorder should be the main goal. In frosted branch response cases, finding and treating the current infectious or autoimmune disorder is our challenge. And for acute idiopathic FBA cases, treating the ocular finding in order to avoid later complications should be our main aim. Thus, we have to keep in mind the characteristic fundus images for each category in order to choose the proper treatment.

\section{Disclosure Statement}

The authors received funding from Angelini farmaceútica ${ }^{\odot}$.

Table 1. Main etiologies related to FBA

Viral agents:

EBV, herpes simplex virus, VZV, CMV, measles, rubella.

Bacterial agents:

Syphilis, tuberculosis, toxoplasmosis, $\beta$-hemolytic streptococcus.

Systemic disorders:

Systemic lupus erythematosus, Crohn's disease, lymphomas, leukemias, multiple sclerosis, Behçet's disease, aseptic meningitis, sarcoidosis. 

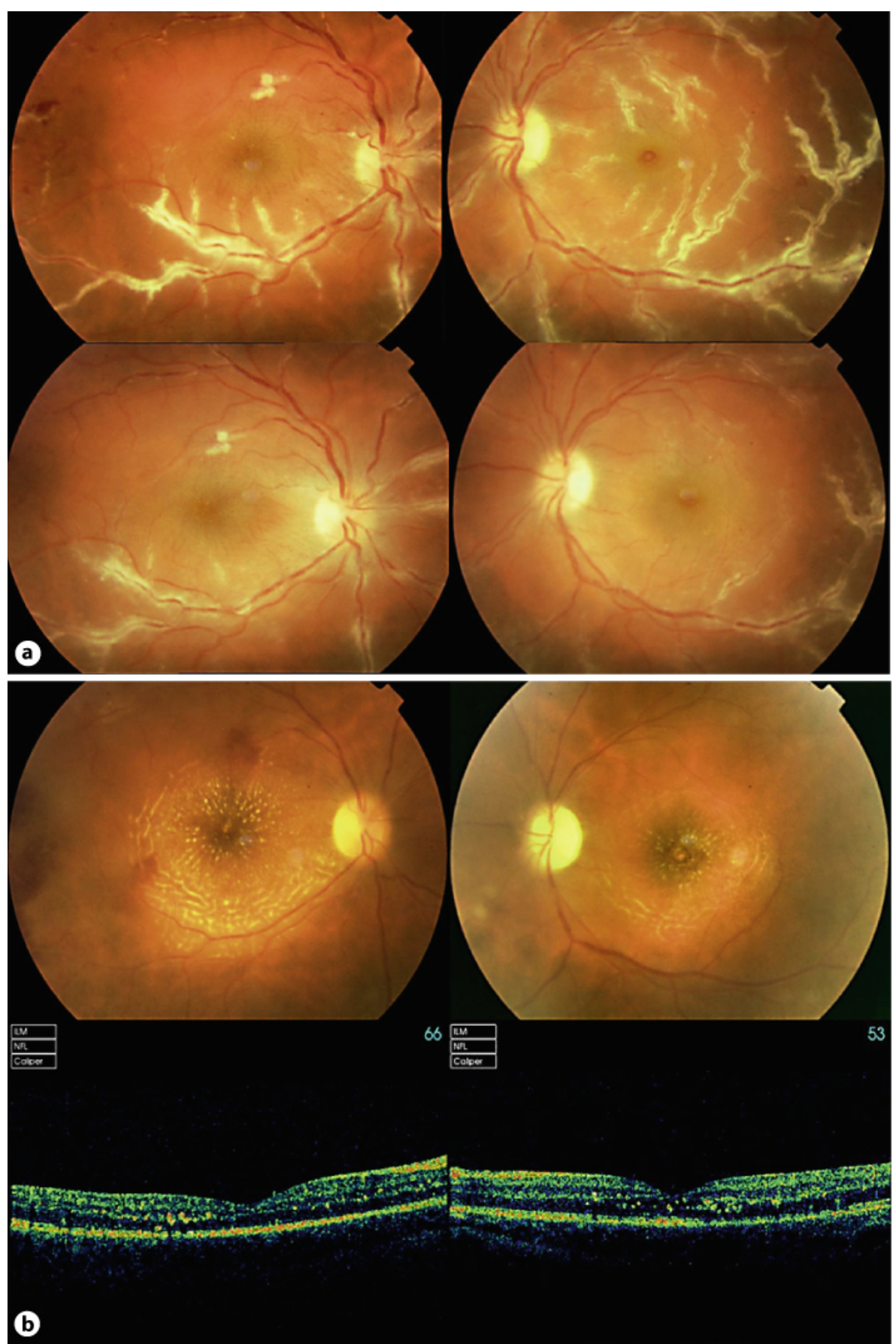

Fig. 1. Both first-line figures show a frosted branch angiitis image before starting with steroids. Both second-line figures disclose a relevant enhancement only $24 \mathrm{~h}$ after starting steroids. Both third-line figures show the macular stars OU, temporal focus of optic nerve atrophy and temporal peripheral hemorrhages 7 days after starting steroids and disclosed with OCT (both last-line figures). 


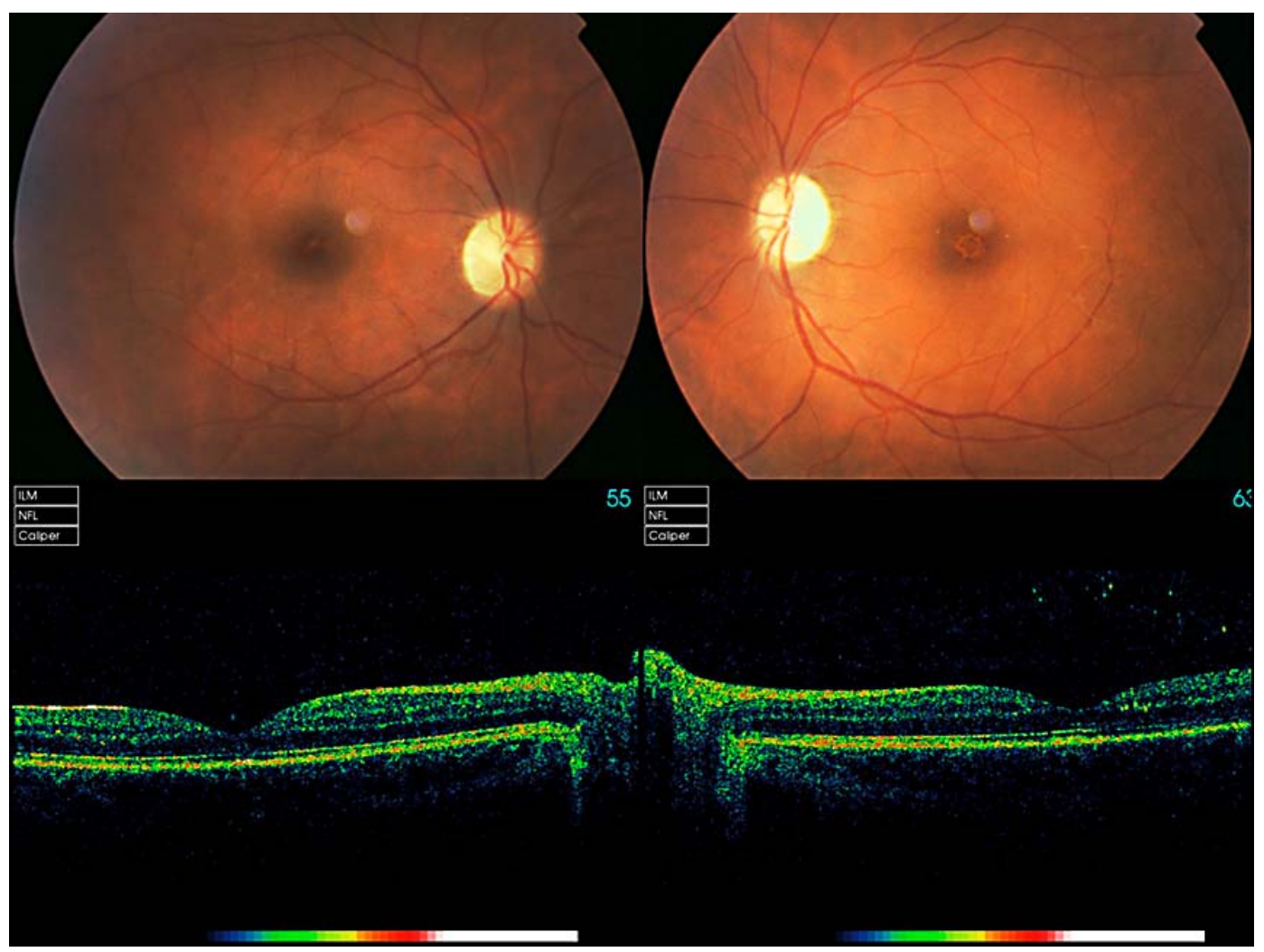

Fig. 2. Fundus and OCT images 4 months after the disorder started. In the upper figures, the retinographies show a mottling pattern at the foveal retinal pigment epithelium level resembling a macular hole which was ruled out with the OCT (both lower figures).

\section{References}

1 Ito Y, Nakano M, Kyu N, et al: Frosted branch angiitis in a child. Jpn J Clin Ophthalmol 1976;30:797-803.

-2 Kleiner RC: Frosted branch angiitis: clinical syndrome or clinical sign? Retina 1997;17:370-372.

-3 Walker S, Iguchi A, Jones NP: Frosted branch angiitis: a review. Eye (Lond). 2004;18:527-533.

4 International Study Group for Behçet’s Disease: Criteria for diagnosis of Behçet's disease. Lancet 1990;335:1078-1080.

5 Reynders S, Dewachter A, de Vriese AS: A case of secondary frosted branch angiitis in Behçet's disease. Bull Soc Belge Ophtalmol 2005;298:41-44.

6 Joseph FG, Scolding NJ: Neuro-Behçet's disease in Caucasians: a study of 22 patients. Eur J Neurol 2007;14:174-180.

7 Nakamura T, Takahashi K, Kishi S: Optic nerve involvement in neuro-Behcet’s disease. Jpn J Ophthalmol 2002;46:100-102.

8 Ideguchi H, Suda A, Takeno M, et al: Neurological manifestations of Behçet's disease in Japan: a study of 54 patients. J Neurol 2010;257:1012-1020.

9 Jackson TE, Pathak S, Doran RM: Behçet disease presenting with frosted branch angiitis. Ocul Immunol Inflamm 2011;19:65-66.

10 Reynders S, Dewachter A, de Vriese AS: A case of secondary frosted branch angiitis in Behçet's disease. Bull Soc Belge Ophtalmol 2005;298:41-44. 